\title{
Efficient Routing through Discretization of Overlapped Road Segments in VANETs
}

\author{
Chao Song, ${ }^{\mathrm{a}, \mathrm{b}, \mathrm{c}}$, Jie $\mathrm{Wu}^{\mathrm{c}}$, Ming Liu ${ }^{\mathrm{a}, \mathrm{b}}$, Huanyang Zheng \\ ${ }^{a}$ School of Computer Science and Engineering, University of Electronic Science and \\ Technology of China, China \\ ${ }^{b}$ Big Data Research Center, University of Electronic Science and Technology of China, \\ China \\ ${ }^{c}$ Department of Computer and Information Sciences, Temple University, US
}

\begin{abstract}
Routing in vehicular ad-hoc networks (VANETs) is based on the contacts through vehicle-to-vehicle communications. Due to the high probability and long duration, contacts on the overlapped trajectories among vehicles play an important role during routing. However, both the stochastic vehicular mobilities and non-ignorable road distances lead to high spatio-temporal fluctuations for the probabilistic contacts on the overlapped trajectories, which can diversify routing decisions. In this paper, we propose a novel Samplingbased Estimation Scheme (SES), which discretizes the probabilistic contacts on the overlapped roads into a small number of segments, and abstracts each segment as a sample. The contact duration between two vehicles moving in opposite directions on their overlapped road is lower, but their contac$\mathrm{t}$ probability is higher. By contrast, the duration of the contact between two vehicles moving in the same direction on their overlapped road is higher, but their contact probability is lower. The proposed SES can achieve efficient routing by considering the above properties of stochastic contacts. Furthermore, we investigate the content transmission among the probabilistic contacts, through the flow model with probabilistic capacities. Extensive experiments validate the competitive performance of the proposed SES with the probabilistic contacts in VANETs.
\end{abstract}

Email addresses: chaosong@uestc.edu.cn (Chao Song), jiewu@temple.edu (Jie $\mathrm{Wu}$, csmliu@uestc.edu.cn (Ming Liu), huanyang.zheng@temple.edu (Huanyang Zheng)

Preprint submitted to Journal of Parallel and Distributed Computing

July 23, 2016

(C) 2016. This manuscript version is made available under the Elsevier user license http://www.elsevier.com/open-access/userlicense/1.0/ 
Keywords: Discretization, overlapped road, probabilistic contact, routing, vehicular ad-hoc networks.

\section{Introduction}

With the increasing demands for various applications on vehicles, both academic researchers and automotive industries have been paying a lot of attention to vehicular ad-hoc networks (VANETs). The vehicle-to-vehicle $(\mathrm{V} 2 \mathrm{~V})$ communications achieve information exchange among vehicles, resulting in an increased range of data dissemination and connectivity to roadside access points (vehicle-to-AP). Many applications have been proposed in VANETs, such as road condition sensing, traffic management, location-based services, and so on [1]. As presented in [2], although the aforementioned services can be supported by wireless infrastructures (e.g., LTE), the corresponding cost is unacceptable. Moreover, these services may be unavailable when such infrastructures do not exist or are damaged. In VANETs, a vehicle first stores the routing message, carries it while moving randomly or on a controlled path, and then forwards it to either an intermediate node or the destination. This is known as a store-carry-forward paradigm [3, 4]. A pairwise V2V contact happens when the two vehicles are within each other's communication range. The places, where the contacts happen among vehicles, are on their overlapped trajectories. These trajectories can be obtained through fixed schedules, navigation systems, and historical trajectory-based predictions $[4,5,6,7]$.

Due to the stochastic vehicular mobilities, the time when a vehicle appears at the vehicles' overlapping trajectories is uncertain, resulting in probabilistic contacts among vehicles. As shown in Figure 1(a), although the trajectories of vehicles $A$ and $B$ are overlapped at intersections 1 and 4 , their contacts at the intersections are uncertain due to their stochastic mobilities. Moreover, even if $A$ and $B$ have contact at the intersection, the contact duration should be short. This is because $A$ and $B$ will leave each other after the intersection. Compared with intersections, the probability of a contact between two vehicles moving in opposite directions on overlapped roads is higher. An example includes the road between intersections 2 and 3 shown in Figure 1(a). On the other hand, the duration of a contact between two vehicles moving in the same direction on the overlapped roads is longer. An example is the road between intersections 2 and 3 shown in Figure 1(b). Therefore, the contact 


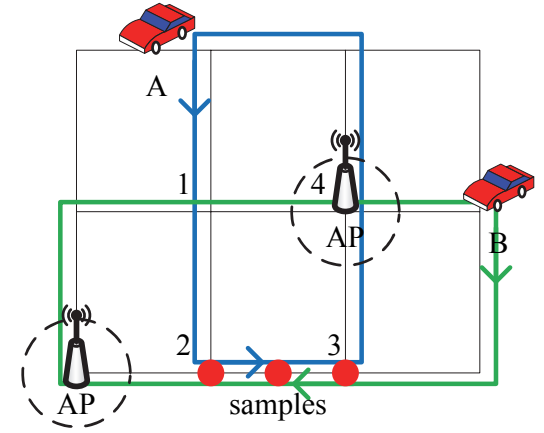

(a) Opposite moving directions

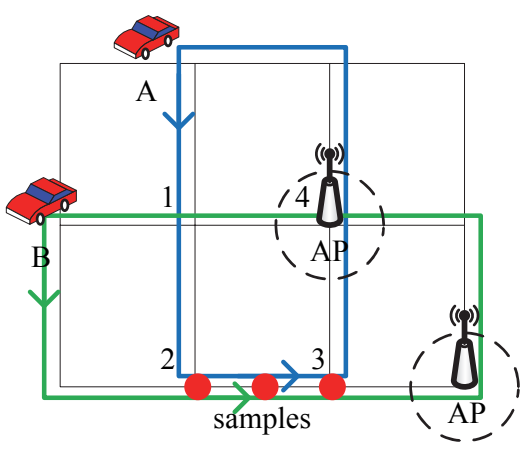

(b) Same moving direction

Figure 1: Probabilistic contact on the overlapped road along the vehicles' trajectories

on the overlapped roads plays an important role in the data delivery among vehicles.

The distance of the overlapped road is vital for making routing decisions, which is non-ignorable. A previous work by Liu and $\mathrm{Wu}[8]$ models the opportunistic routing as a probabilistic state-space graph, and discretizes each probabilistic contact between two nodes as a point associated with a time slot and a contact probability. Since the distance of the overlapped road is non-ignorable, the probabilistic contact on this road has high spatiotemporal fluctuation, which provides more possible routing paths. Let us consider the scenario in Figure 1(a), where $A$ wants to send a message to an AP as soon as possible. If $A$ meets $B$ near intersection 2, it will forward the message to $B$, which has a shorter travel distance to an AP. In contrast, if $A$ meets $B$ near intersection 3, it will keep on carrying the message to a nearer AP.

In this paper, we study the probabilistic contacts in the DieselNet trace [9], and discuss stochastic vehicular mobility model. Then, by involving the fluctuation of the contacts on the road with non-ignorable distance, we propose a Sampling-based Estimation Scheme (SES) for routing with probabilistic contacts on the overlapped roads. The basic idea is that we discretize the probabilistic contacts on the overlapped road into a small number of segments, and abstract each segment as a sample, such as the red points in Figure 1. Different from the one-point solution in [8], each sample provides a possible routing decision on the overlapped road, and all the samples together can involve all the possible routing paths with different distance informa- 
tion. Meanwhile, SES filters the illegible samples by the spatio-temporal constraints of contacts on the overlapped roads. Note that more samples bring a higher accuracy of the delay estimation, as well as a higher computational cost. Therefore, we will discuss the number of samples (i.e. discretization level) in order to leverage the tradeoff between the accuracy and the cost.

Furthermore, the probabilistic contact durations have significant impacts on the throughput between two inter-contact vehicles. Consequently, we investigate maximum content transmission among the probabilistic contacts, under a flow model with probabilistic capacities. Since the maximum flow problem under probabilistic capacities is NP-hard [10], we propose a heuristic algorithm to approximate the maximum flow for a reliable content transmission. We conduct intensive simulations for discussing the data forwarding with the probabilistic contacts on the overlapped roads in VANETs, which are based on the combination of NS-2, SUMO, and MOVE $[11,12,13]$. The simulation results imply that an accurate estimation for the probabilistic contacts can affect the performance of contact-based data delivery in VANETs.

Our technical contributions are multi-fold, including:

- We propose a Sampling-based Estimation Scheme (SES) for routing with probabilistic contacts on the overlapped roads, in order to increase the accuracy of the delay estimation. It discretizes the probabilistic contacts on the overlapped road into a small number of segments.

- Due to the probabilistic contact durations on the overlapped roads, we investigate maximum flow among the probabilistic contacts for the content transmission, through the flow model with probabilistic capacities.

- By the combination of NS-2, SUMO, and MOVE, we conduct intensive simulations for evaluating the performance of SES with the probabilistic contacts in VANETs.

This paper is organized as follows: Section 2 introduces the assumptions and analyzes probabilistic contacts. Section 3 proposes the Sampling-based Estimation Scheme (SES). Section 4 investigates the content transmission with the probabilistic contacts. In Section 5, we describe the results from our simulations. In Section 6, we discuss related work in this area. Finally, in the last section, we conclude the paper. 
Table 1: Notations

\begin{tabular}{|c||l|}
\hline Notation & Description \\
\hline \hline$r_{i j}$ & The road between the intersection $i$ and $j$. \\
\hline$t_{i j}$ & The travel time from the place point $i$ to $j$. \\
\hline$l_{i j}$ & The Euclidean distance from the place point $i$ to $j$. \\
\hline$v$ & The speed of a vehicle. \\
\hline$R$ & Communication range of the vehicle node. \\
\hline$A B$ & The contact between the vehicle $A$ and $B$. \\
\hline $\mathcal{T}_{A B}$ & The starting time of the contact $A B$. \\
\hline $\mathcal{P}_{A B}$ & The probability of the contact $A B$. \\
\hline $\mathcal{D}_{A B}$ & The duration of the contact $A B$. \\
\hline
\end{tabular}

\section{Probabilistic Contact}

In this section, we first give the assumptions and definitions. We analyze a real trace, and then set up a model of the probabilistic contacts.

\subsection{Assumptions and Definitions}

Notations used in this paper are listed in Table 1. We assume that each vehicle knows its location by the GPS service, which is already available in most new cars, and will be common in the future. Vehicles can obtain the trajectories of others by some existing approaches: (1) The vehicles have fixed routes (such as buses) [9], which can be obtained by their schedules [5]; (2) The trace of a node can be obtained through the navigation system equipped onboard in the vehicle [6]; (3) Due to the spatio-temporal regularity with vehicle mobility, the future contacts and trajectories of vehicles can be predicted $[4,7]$. We assume the speed of vehicles (denoted by $v$ ) in the mobility model follows a Gaussian distribution.

Vehicles communicate with each other through short-range wireless channels. Let $R$ denote the communication range of each vehicle node. Here, we make the assumption that two vehicles would have a contact opportunity if their locations are within the communication range. For simplification, we assume each vehicle can communicate with only one other vehicle on the same road, due to the obstacles (such as the buildings) on the roadside. The V2V contact between vehicles $A$ and $B$ is denoted by $A B$. We use the probability, starting time, and duration to describe such contact.

Definition 1 (Probability of Contact). We define probability of contact as the chance that a vehicle can encounter another vehicle on their overlapped road. Let $\mathcal{P}_{a b}$ denote the probability of contact between nodes a and $b$. 


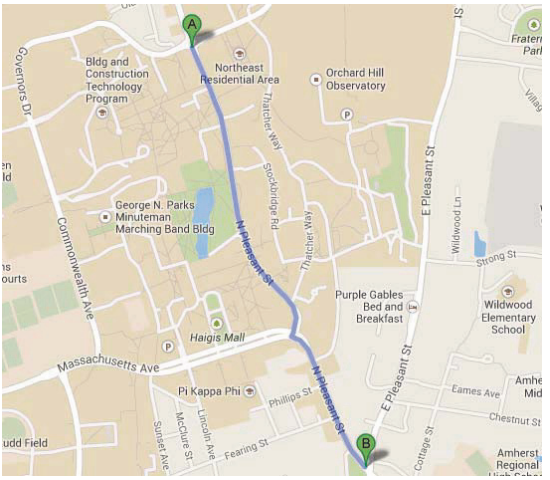

(a) Overlapped road segment

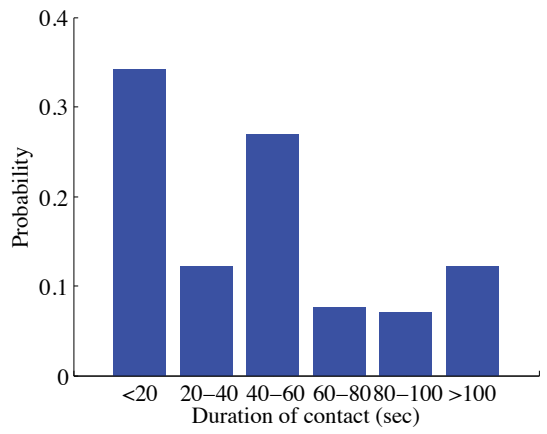

(b) Duration of contact

Figure 2: Probabilistic contacts on the overlapped road in DieselNet

Definition 2 (Starting Time of Contact). We define the starting time of a contact as the time slot when a vehicle contacts another one. We simply assume the initial time is 0. Let $\mathcal{T}_{a b}$ denote the starting time of contact between nodes $a$ and $b$.

Definition 3 (Contact Duration). The contact duration is the total time that a couple of vehicles are within communication range of one another. Let $\mathcal{D}_{a b}$ denote the duration of contact between nodes $a$ and $b$.

The relationship between routing and content transmission is the probabilistic contact (Definition 1). In this paper, we investigate not only its time (Definition 2) for estimate delay, but also its duration (Definition 3) for estimate throughput.

\subsection{Analysis with DieselNet Trace}

We investigate the probabilistic contacts with the trace from DieselNet [9]. The trace records the contacts among the buses in UMass, which includes the starting time, position, and duration of each contact. We choose the trace of the bus with ID 3007, and analyze its contacts with the buses on route 31 along N Pleasant Street, as shown in Figure 2(a). During each occasion of traveling on the street, we sum the durations of the contacts with each other. For example, during one travel on the street, there are three records of contacts with bus 3118 , whose durations are $7.63,3.38$, and 13.77 seconds; so the total duration of the contact for this travel on the street between buses 
3007 and 3118 is 24.78 seconds. Thus, we obtain the contact durations of bus 3007 with other buses. The probability of contact durations is shown in Figure 2(b).

We notice that the probability of the short duration, which is less than 20 seconds, has the highest probability (about 0.35). Short durations of contact may be caused by the buses with different directions on the road or at an intersection. The probability of the duration being between 20 and 100 seconds is about 0.53 , which may be caused by buses heading in the same direction on the road. The duration of the contact occurring in the same moving direction is related to their travel time spent together on the mutual overlapped road. Studies on traffic engineering have demonstrated that the travel time of a vehicle along a fixed distance follows the Gamma distribution [14]. We notice that between 20 and 100 seconds of duration, the probability of duration between 40 and 60 seconds is the highest (about 0.27). The probability of long duration, which is more than 100 seconds, is about 0.12 . This may result from the long waiting time at a bus stop.

\subsection{Model of Probabilistic Contact}

Traffic engineering has widely agreed that the speed of vehicles follows a Gaussian distribution $[15,16]$, i.e., $v \sim N\left(\mu_{v}, \sigma_{v}^{2}\right)$. Due to the stochastic vehicular mobilities, the time when a vehicle appears at the vehicles' overlapping trajectories is uncertain, resulting in probabilistic contacts among vehicles. We discuss the probabilistic contacts under the two situations shown in Figure 1.

First, let's consider the situation that the vehicle $A$ may meet $B$ on their mutual overlapped road $r_{i j}$ with the opposite moving directions, such as the road $r_{23}$ in Figure $1(\mathrm{a})$. Let $l_{A}\left(t, r_{i j}\right)$ and $l_{B}\left(t, r_{i j}\right)$ denote their travel distances on the road $r_{i j}$ at time $t$, respectively. We assume the travel distances of two vehicles at a given time are independent from each other. If $A$ contacts $B$ with the opposite moving direction on the road $r_{i j}$, then the sum of their travel distances on the road before the contact is equal to the length of the road (the length of $r_{i j}$ is denoted by $l_{i j}$ ) minus the communication range (denoted by $R$ ). Let $p_{A B}(t, x)$ denote the probability distribution function of the contact $A B$ at time $t$, where the travel distance of $A$ on the road $r_{i j}$ is $x\left(0 \leq x \leq l_{i j}\right)$. Thus, we have:

$$
p_{A B}(t, x)=P\left[l_{A}\left(t, r_{i j}\right)=x\right] \cdot P\left[l_{B}\left(t, r_{i j}\right)=l_{i j}-R-x\right]
$$

where $P[X]$ denotes the probability for $X$ to be true. 
Due to the stochastic vehicular mobility, the contact $A B$ with opposite moving directions on the overlapped road $r_{i j}$ can happen at any place on the road. The probability of this contact can be calculated as:

$$
\mathcal{P}_{A B}=\int_{0}^{\infty} \int_{0}^{l_{i j}} p_{A B}(t, x) d x d t
$$

The expected starting time of the contact $A B$ on the road $r_{i j}$ can be calculated as:

$$
E\left[\mathcal{T}_{A B}\right]=\left(1 / \mathcal{P}_{A B}\right) \cdot \int_{0}^{\infty} \int_{0}^{l_{i j}} p_{A B}(t, x) \cdot t d x d t
$$

where the normalization coefficient $1 / \mathcal{P}_{A B}$ means that the expected value is calculated under the conditional event of contact.

The duration of the contact $A B$ with opposite moving directions is from the starting time of the contact to the time when one of them moves out of the road, or to another's communication range. For approximate calculation, we assume that the speed of $A$ is the same as $B$ during their contact (denoted by $v$ ), and the contact at the ends of the overlapped road is ignored. Thus, the contact duration of vehicles with opposite directions can be approximately calculated by:

$$
\mathcal{D}_{A B}=R / v
$$

Let's consider another situation that the vehicle $A$ may meet $B$ on their mutual overlapped road $r_{i j}$ with the same moving direction, such as the road $r_{23}$ in Figure 1(b). Since $A$ and $B$ have the same speeds, the contact $A B$ on the road $r_{i j}$ should be satisfied that, when the later vehicle moves onto the overlapped road, the travel distance of another vehicle on the road is less than the communication range $R$. When they start to contact each other, their travel distances on the overlapped road $r_{i j}$ should be satisfied $0 \leq l_{A}\left(t, r_{i j}\right), l_{B}\left(t, r_{i j}\right) \leq R$. Thus, the probability distribution function of the contact at time $t$, where the travel distance of early vehicle is equal to $x$ $(0 \leq x \leq R)$, can be calculated as follows:

$$
\begin{aligned}
p_{A B}(t, x) & =P\left[l_{A}\left(t, r_{i j}\right)=0\right] \cdot P\left[l_{B}\left(t, r_{i j}\right)=x\right] \\
& +P\left[l_{B}\left(t, r_{i j}\right)=0\right] \cdot P\left[l_{A}\left(t, r_{i j}\right)=x\right] \\
& -P\left[l_{A}\left(t, r_{i j}\right)=0\right] \cdot P\left[l_{B}\left(t, r_{i j}\right)=0\right] .
\end{aligned}
$$

However, while these two vehicles $A$ and $B$, with the same moving direction, meet on their overlapped road, the distance from the contact point to 
the entrance of the road should be less than their communication range. The probability of the contact $A B$ can be calculated as:

$$
\mathcal{P}_{A B}=\int_{0}^{\infty} \int_{0}^{R} p_{A B}(t, x) d x d t
$$

The expected starting time of the contact $A B$ on the road $r_{i j}\left(t_{c}\right)$ can be calculated as:

$$
E\left[\mathcal{T}_{A B}\right]=\left(1 / \mathcal{P}_{A B}\right) \cdot \int_{0}^{\infty} \int_{0}^{R} p_{A B}(t, x) \cdot t d x d t
$$

The duration of the contact $A B$ with the same moving speed is from the starting time of the contact to the time when one of them moves out of the road. Thus, the road distance increases the contact duration, and the expected duration of contact $A B$ on the overlapped road $l_{i j}$ can be calculated as follows:

$$
E\left[\mathcal{D}_{A B}\right]=\left(1 / \mathcal{P}_{A B}\right) \cdot \int_{0}^{\infty} \int_{0}^{l_{i j}} \frac{l_{i j}-x}{v} \cdot p_{A B}(t, x) d x d t .
$$

Based on the analysis of the probabilistic contacts with the stochastic mobility model, we notice that the road distance increases both the probability and the duration of the contact between vehicles. Therefore, the contacts on the overlapped roads plays an important role in routing among vehicles.

\section{Sampling-based Estimation Scheme}

In this section, we show an overview of the routing scheme, the objective of which is the expected minimum delay. By considering the temporal and spacial probabilistic contacts on the overlapped roads, we propose a Sampling-based Estimation Scheme (SES) for evaluating the data delivery among the probabilistic contacts with high accuracy. Furthermore, in order to increase the accuracy of estimation, we generalize SES to use $k$ samples.

\subsection{Probabilistic State-space Graph}

We utilize a metric of expected minimum delay (EMD) [8] to evaluate the data delivery among the probabilistic contacts as follows: 


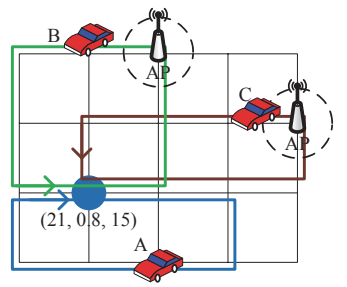

(a) Scenario

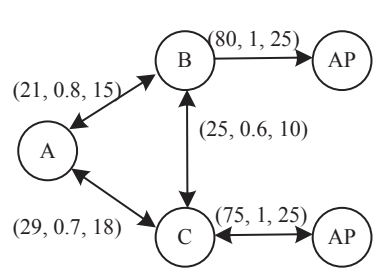

(b) Evolving graph $\mathcal{G}$

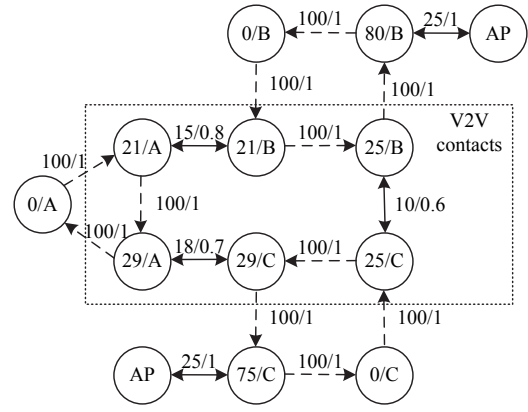

(c) Probabilistic state-space graph $G$

Figure 3: An example of three vehicles with cyclic trajectories

Definition 4 (Expected Minimum Delay). The expected minimum delay is the expected time an optimal opportunistic forwarding scheme takes to deliver a message, given a starting time and a source-destination pair.

We abstract each contact between pairwise vehicles as a point on their overlaps. This point is denoted as a tuple of $(\mathcal{T}, \mathcal{P}, \mathcal{D})$, where $\mathcal{T}, \mathcal{P}$, and $\mathcal{D}$ denote the starting time, probability, and duration of the contact, respectively. A simple example of three vehicles $(A, B$, and $C)$ is shown in Figure $3(\mathrm{a})$, which move along the cyclic trajectories at different speeds. Although their mobilities are stochastic, we assume they can be re-synchronized at their beginning points by the waiting time (such as buses in city [5]), and the duration of the cycle is 100 time slots. The vehicle $A$ wants to send a message to an AP, which can be met by vehicles $B$ and $C$. We assume $B$ and $C$ connect to an $\mathrm{AP}$ at the $80^{\text {th }}$ and $75^{\text {th }}$ time slots, while the durations of the connections are 25 time slots. Through statistics or mobility prediction, the contacts are listed in Table 2. The starting time in the table lists its variation, for example, 10-32 means the variation of the starting time of the contact $A B$ is from 10 to 32 time slots. Thus, the expected value of the contacts of $A B, B C, C A$ are $(21,0.8,15),(25,0.6,10)$, and $(29,0.7,18)$, respectively. We use an evolving graph [17] to describe the contacts among the three nodes, which captures the dynamic characteristics of each node and link, as follows:

Definition 5 (Evolving Graph). Let us be given a graph $\mathcal{G}^{\prime}(\mathcal{V}, \mathcal{E})$ and an ordered sequence of its subgraphs, $S_{\mathcal{G}^{\prime}}=\mathcal{G}_{1}^{\prime}, \mathcal{G}_{2}^{\prime}, \cdots, \mathcal{G}_{T}^{\prime}$ such that $\cup_{i=1}^{T} \mathcal{G}_{i}^{\prime}=\mathcal{G}^{\prime}$. 
Table 2: An example of the probabilistic contacts

\begin{tabular}{|c|c|c|c|}
\hline Contact & Starting time $(\mathcal{T})$ & Probability $(\mathcal{P})$ & Duration $(\mathcal{D})$ \\
\hline$A B$ & $10-32$ & 0.8 & 15 \\
$B C$ & $20-30$ & 0.6 & 10 \\
$C A$ & $18-40$ & 0.7 & 18 \\
\hline
\end{tabular}

Let $S_{T}=t_{0}, t_{1}, t_{2}, \cdots, t_{T}$ be an ordered sequence of time instants. Then the system $\mathcal{G}=\left(\mathcal{G}^{\prime}, S_{\mathcal{G}}^{\prime}, S_{T}\right)$, where each $\mathcal{G}_{i}^{\prime}$ is the subgraph in place during $\left[t_{i-1}, t_{i}\right)$, and is called an evolving graph. Let $V_{\mathcal{G}}=\mathcal{V}$ and $E_{\mathcal{G}}=\mathcal{E}$.

Figure 3(b) shows the evolving graph $(\mathcal{G})$ of the given example, and each edge has a weight with the tuple of $(\mathcal{T}, \mathcal{P}, \mathcal{D})$ for describing the contact between the two vertices.

In order to remove the time dimension from the evolving graph $\mathcal{G}$, we generate a probabilistic state-space graph $G=(V, E)$, where $V$ is the set of states and $E$ is the set of links which are time-independent, as shown in Figure 3(c). For each vehicle $u$, we create a set of states $\left\{t_{i} / u\right\}$ in $G$ for each time slot $t_{i}$ when the vehicle $u$ has one or more discretized probabilistic contacts. For example, three states $0 / A, 21 / A$ and $29 / A$ are created in $G$ for the vehicle $A$. There are two types of links in $G$ : directional link and bidirectional link. The directional link connects the consecutive states of a node into a ring. The bidirectional link in $G$ is created corresponding to each discretized probabilistic contact. Different from the graph introduced in [8], we use $c / p$ for labeling both types of links, where $c$ is the capacity and $p$ is the probability of the transition. We consider a simple wireless communication model with the bandwidth of $1 \mathrm{Mb}$ per time slot, and the buffer size of each node is $100 \mathrm{Mb}$. For example, the link of the contact between $A$ and $B$ is $15 / 0.8$, which means its capacity is $15 \mathrm{Mb}$ with the probability of 0.8 .

However, the approach in [8] abstracts the contact on each overlapped road as a point, and thus, the contacts among the vehicles have only one temporal sequence. Take the example in Figure 3(c): the temporal sequence of the contacts is $A B \rightarrow B C \rightarrow C A$. However, as listed in Table 2, due to the probabilistic contacts on the overlapped roads, the starting time of each contact is varied, and the temporal sequence of the contacts is not fixed. For example, consider when the starting time of the contacts $A B, B C$, and $C A$ are 32,20 , and 18 time slots, respectively, and the temporal sequence changes as: $C A \rightarrow B C \rightarrow A B$. Therefore, the drawbacks of this approach are that it misses some available routing paths under different temporal sequences of contacts. On the other hand, as aforementioned in the discussion of Figure 


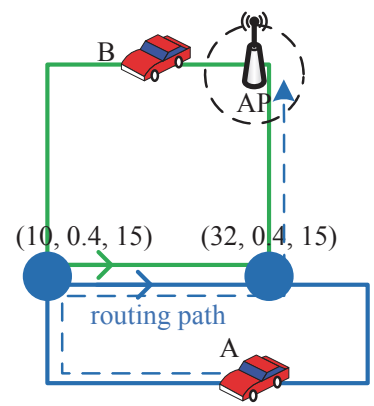

(a) Two samples for $A B$

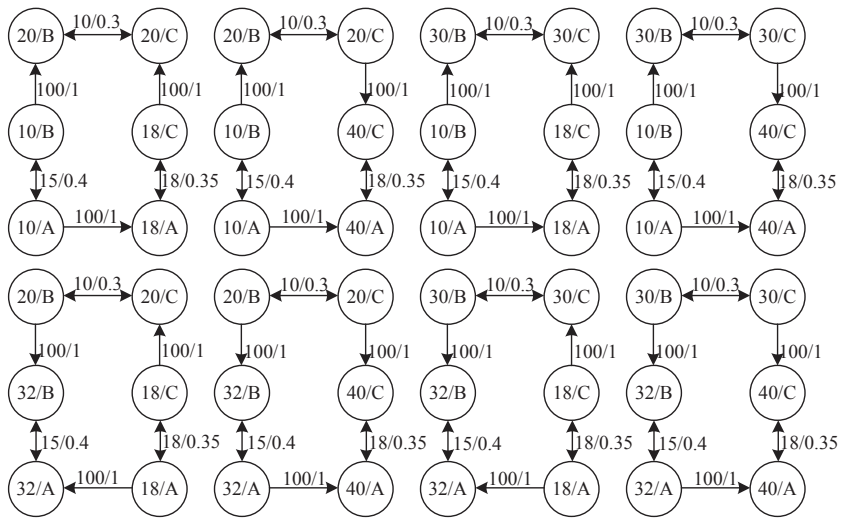

(b) The 8 possible state-space graphs

Figure 4: V2V contacts with two samples

1(a) in Section 1, the routing is different when two vehicles contact each other at different places on their overlapped road. In the next subsection, we will introduce a novel approach for estimating EMD by considering the temporal and spacial probabilistic contacts on the overlapped roads.

\subsection{Discretization with Two Samples}

As in the aforementioned discussion, the approach used in [8] discretizes each contact as a point. Take the example of the probabilistic contact between $A$ and $B$ on an overlapped road, as shown in Figure 3(a); we discretize the contact as a point in the middle of the overlapped road. We denote it as a sample of the probabilistic contact. The sample of the contact between the vehicles $A$ and $B$ is denoted by $s_{A B}(\mathcal{T}, \mathcal{P}, \mathcal{D})$, where $\mathcal{T}, \mathcal{P}$, and $\mathcal{D}$ denote the starting time, probability, and duration, respectively. As shown in Figure $3(\mathrm{c})$, if we only generate one sample for each contact, then we may miss some possible routing paths.

To solve the above problem, we propose a Sampling-based Estimation Scheme by spatio-temporally discretizing the probabilistic contacts on the overlapped roads. Figure 4(a) shows an example of SES with two samples. The two samples for the probabilistic contact on the overlapped road are at both ends of the road, which are denoted by $s_{A B}^{(1)}\left(\mathcal{T}_{A B}^{(1)}, \mathcal{P}_{A B}^{(1)}, \mathcal{D}_{A B}^{(1)}\right)$ and $s_{A B}^{(2)}\left(\mathcal{T}_{A B}^{(2)}, \mathcal{P}_{A B}^{(2)}, \mathcal{D}_{A B}^{(2)}\right)$ for the contact $A B$. Since $A$ carries the message onto the overlapped road and contacts with $B$, the starting time of the sample at 


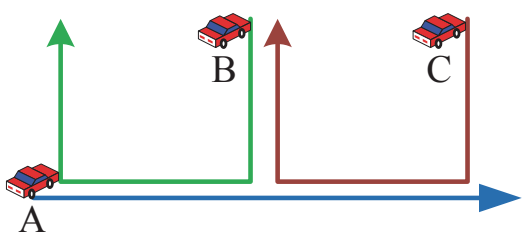

(a) Spacial sequence constraint

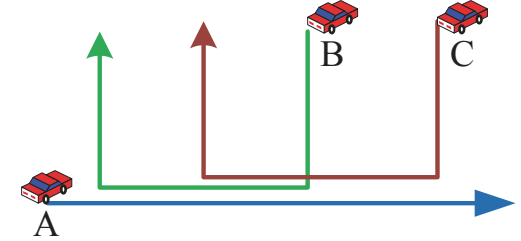

(b) Contact sequence constraint

Figure 5: Constraint of contact sequence

the entrance of the road along $A$ 's trajectory is set as the minimal starting time of $A B$; the starting time at the exit of the road is the maximum. Here, we simply assume the probability of contact $A B$ is uniformly distributed on the overlapped road, so the probabilities of the two samples are the half of the probability of $A B$. In the next subsection, we will discuss the allocation of contact probability with non-uniform distribution. The duration of each sample is equal to the expected duration of the contact. Thus, the two samples in the example are $s_{A B}^{(1)}(10,0.4,15)$, and $s_{A B}^{(2)}(32,0.4,15)$, respectively.

By combining the samples for each contact, we can obtain $2^{n}$ state-space graphs with different states of nodes and directions of edges, where $n$ denotes the number of vehicles. As in the aforementioned example, the two samples of each contact are as follows: $s_{A B}^{(1)}(10,0.4,15), s_{A B}^{(2)}(32,0.4,15), s_{B C}^{(1)}(20,0.3,10)$, $s_{B C}^{(2)}(30,0.3,10), s_{C A}^{(1)}(18,0.35,18)$, and $s_{C A}^{(2)}(40,0.35,18)$. Figure 4(b) shows the obtained eight $\left(2^{3}\right)$ state-space graphs with two samples. We notice that different graphs have different states of nodes, i.e., starting times of the contacts. Since the direction of the edge between two contacts is from the early one to the later one, the states of nodes result in different directions of edges in these graphs.

By considering the moving directions and overlapped roads of the vehicles, a potential problem is that some illegible state-space graphs need to be filtered. As in the example of the contacts $A B, B C$, and $C A$ with different contact times, there are six possible sequences among the contacts. Figure 5 (a) shows an example where the three pairwise contacts $(A B, B C$, and $C A$ ) are spatially independent. We assume there exists an temporal overlap of starting time among the contacts, due to fluctuation of the probabilistic contacts. By considering the moving direction of each vehicle, their contacts should be satisfied: $\mathcal{T}_{A B}<\mathcal{T}_{A C}, \mathcal{T}_{B C}<\mathcal{T}_{B A}$, and $\mathcal{T}_{C A}<\mathcal{T}_{C B}$. Figure 5(b) shows another example where the trajectories of three vehicles have a mutual 


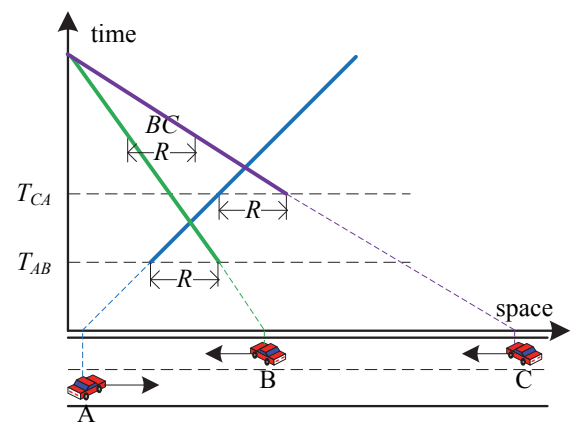

Figure 6: Space-time graph of three vehicles on the mutual overlapped road

overlapped road. We can filter some illegible sequences of contact events on the mutual overlapped road with a space-time graph, which is widely used in traffic engineering. We assume the moving directions of the three vehicles $A$, $B$, and $C$ on their mutual overlapped roads are known, and that the speed of $C$ is larger than that of $B$, which means $C$ can catch up with $B$. Given two pairwise contact times (e.g. $A B, C A$ ), we can obtain the legible contact sequence with the third contact (e.g. $B C$ ) by a space-time graph, as shown in Figure 6. In the graph, the horizontal axis denotes the space of the road, and the vertical axis denotes the time. Thus, a point $(x, y)$ in the graph denotes that the position of a vehicle is at $x$ on the road when the time is $y$, and each line denotes the mobility of a vehicle with a speed as its slope. When the horizontal distance between two lines is less than the communication range $R$, a contact between the two vehicles happens. By a given $\mathcal{T}_{A B}$ and $\mathcal{T}_{C A}$, we can obtain the lines of the three vehicles. Thus, the legible sequence of contacts is determined, i.e. the contact time between $B$ and $C$ should be satisfied: $\mathcal{T}_{B C}>\mathcal{T}_{C A}>\mathcal{T}_{A B}$. Then, we can filter the illegible sequences of contact events.

The expected delays of the nodes in each state-space graph can be calculated by the model proposed in [8]. The probability of each state-space graph is equal to the joint probability of the probabilistic contacts in the graph, and we normalize the probabilities of all legible state-space graphs. Based on the expected delay of nodes in each state-space graph, and the normalized probability of each graph, we can obtain the expected delay of each probabilistic contact in the networks for calculating the routing path by the shortest path algorithm. 


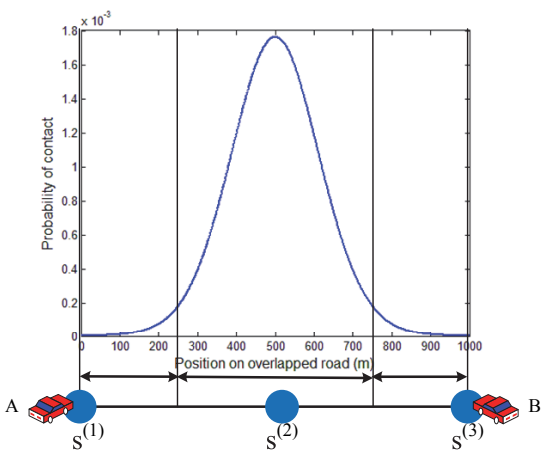

(a) $k=3$

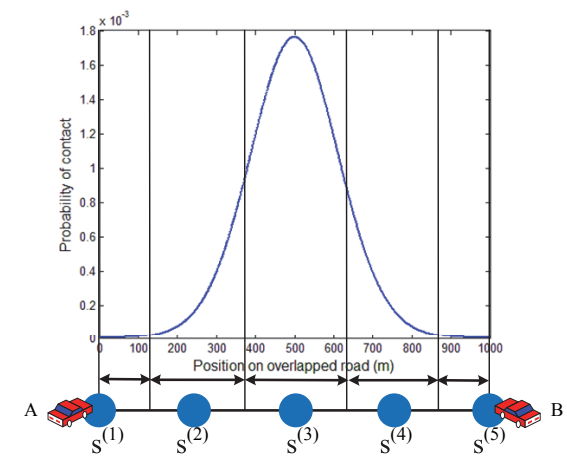

(b) $k=5$

Figure 7: Assignment of weights with $k$ samples

\subsection{Discretization with $k$ Samples}

In this subsection, we discuss the discretization of SES with $k(k \geq 2)$ samples on an overlapped road. In the scheme with $k$ samples, the positions of two samples are at each end of the road for involving all possible routing paths, and the rest of samples are uniformly distributed on the road to increasing its accuracy. The distance between two adjacent samples is equal to $l_{i j} /(k-1)$, where $l_{i j}$ is the length of the road $r_{i j}$.

Take three samples as an example (see in Figure 7(a)), two samples are at two ends of the road, and the remaining sample is at the middle of the road. For five samples as shown in Figure 7(b), two samples are at the ends of the road, and the rest of three samples are uniformly distributed on the road. As discussed in discretization with two samples, since the message is delivered from one vehicle (e.g. A) to another (e.g. B) on their overlapped road, the starting time of the sample at the entrance of the road along $A$ 's trajectory is set as the minimal starting time of $A B$; the starting time at the exit of the road is the maximum. Thus, the starting time of the $k$ samples are also uniformly distributed in the range of the starting time of contact between the vehicles $A$ and $B$, which is denoted by $\left[\mathcal{T}_{\text {min }}, \mathcal{T}_{\text {max }}\right]$. Thus, the starting time of the $m^{\text {th }}(1 \leq m \leq k)$ sample $s_{A B}^{(m)}$ is calculated as: $\mathcal{T}_{A B}^{(m)}=\mathcal{T}_{\text {min }}+\left(\mathcal{T}_{\text {max }}-\mathcal{T}_{\text {min }}\right) \cdot \frac{m-1}{k-1}$. We set the place of a boundary between two adjacent samples in the middle of them, such as the solid lines in Figures 7(a) and 7(b), and each end of the road also has a boundary. Thus, the probability of contact for each sample $\mathcal{P}_{A B}^{(m)}$ is the cumulative probability between its two adjacent boundaries. The contact duration of each sample 


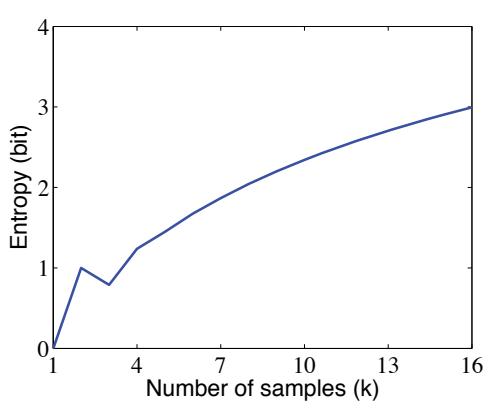

(a) $H(A B)$

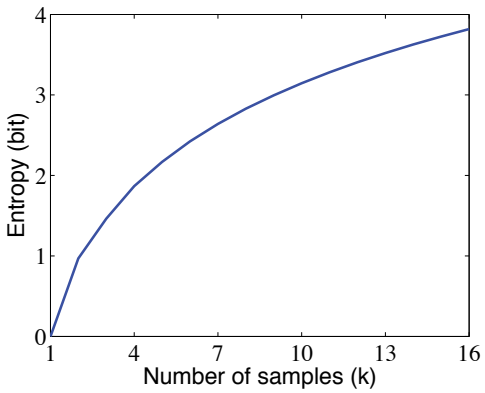

(b) $H(B C \mid A B)$

Figure 8: Entropy of sample-based contacts on the overlapped roads

is equal to the expected duration of the contact on the road $\mathcal{D}_{A B}$.

To determine a feasible $k$, we look into the information theory, where entropy is a measurement of the uncertainty in a random variable. The term usually refers to the Shannon entropy, which quantifies the expected value of the information contained. Thus, we examine the information contained in the probabilities of contacts with $k$ samples, by computing the entropy and conditional entropy of multiple contacts. We use the mobility model introduced in Section 2.3. As in the aforementioned discussion, the entropy of the probability of contact $A B$ can be calculated as follows:

$$
H(A B)=-\sum_{i=1}^{k} \mathcal{P}_{A B}^{(i)} \cdot \log _{2} \mathcal{P}_{A B}^{(i)}
$$

Figure 8(a) shows the entropy of an overlapped road between two sequential contacts. We notice that the entropy is increasing with the number of samples $(k \geq 3)$. This is because a large number of samples includes the data delivery delay at different positions of contact on the overlapped road. However, the entropy when $k=2$ is larger than that when $k=3$, since the speed of vehicles in the mobility model follows a Gaussian distribution. The joint entropy of the probability of contacts $A B$ and $B C$ on the two sequential overlapped roads is:

$$
H(A B, B C)=-\sum_{0 \leq i, j \leq k}\left[\mathcal{P}_{A B}^{(i)} \cdot \mathcal{P}_{B C}^{(j)}\right] \cdot \log _{2}\left[\mathcal{P}_{A B}^{(i)} \cdot \mathcal{P}_{B C}^{(j)}\right]
$$

where $\mathcal{P}_{A B}^{(i)} \cdot \mathcal{P}_{B C}^{(j)}$ is the joint probability for the $i^{\text {th }}$ sample of contact $A B$ and the $j^{\text {th }}$ sample of contact $B C$, which are assumed to be independent of each 


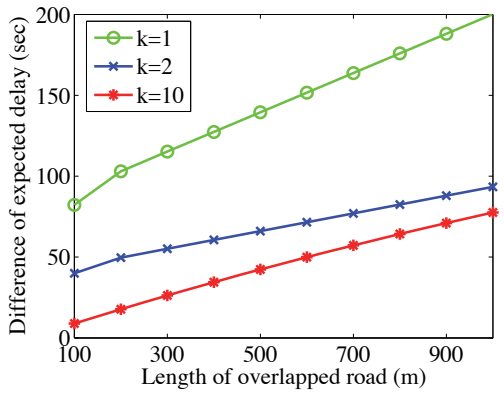

(a) Short road

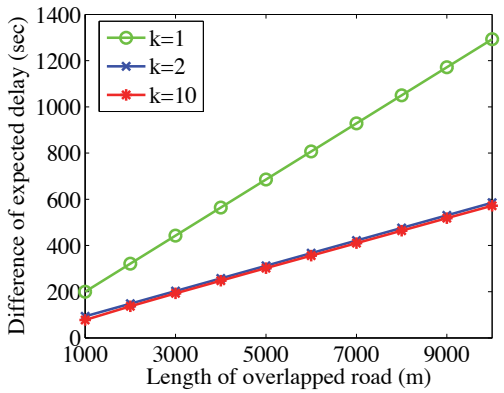

(b) Long road

Figure 9: Expected delivery delay with different numbers of samples $(k)$

other. With $H(A B)$ and $H(A B, B C)$, the conditional entropy of contact of $B C$ with $A B$ can be calculated as follows:

$$
H(B C \mid A B)=H(A B, B C)-H(A B) .
$$

Figure 8(b) shows the conditional entropy of the contact $B C$. We notice that the entropy increases exactly while $k$ is increasing, which means a larger number of samples can involve more information for routing.

Let us consider the scenario in Figure 1(a), the vehicles move with the mobility model introduced in Section 2.3. Figure 9 shows the difference of expected delays obtained by $k$ samples under different road lengths. The results include short road from 100 to 1,000m, shown in Figure 9(a), and long road from 1,000 to $10,000 \mathrm{~m}$, shown in Figure 9(b). We notice that the expected delay obtained by 2 samples is much closer to the delay obtained by 10 samples. Because all contacts in the networks have $k$ samples by SES, the number of state-space graphs with $n$ vehicle contacts is $k^{n}$. A large number of samples $k$ can result in a state explosion problem. Therefore, by considering the trade-off between accuracy and computational cost, we adopt two samples in our sampling-based estimation scheme.

\section{Content Transmission with Probabilistic Contacts}

Due to the probabilistic contacts on the overlapped roads, the contact duration between two vehicles moving in the opposite directions on their overlapped road is lower, but with higher probability of contact; meanwhile, the duration of the contact between two vehicles moving in the same direction 


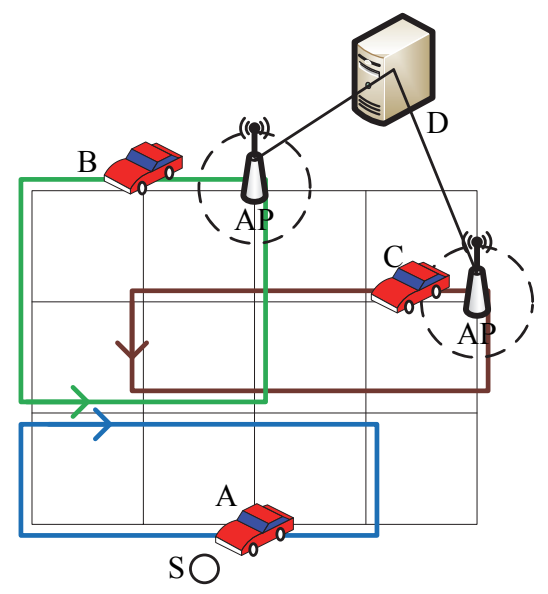

Figure 10: An example of content transmission

on their overlapped road is higher, but with lower probability of contact. By utilizing the contact duration, we investigate the content transmission based on the probabilistic contacts on the overlapping roads.

\subsection{Problem of Content Transmission}

As shown in Figure 10, the source node $S$, which can be a vehicle or a road side unit (RSU), has a content file to send to the destination $D$. For efficient transmission, the content file is divided into several packets with fixed sizes. The contact between two vehicles has a probability, a duration, and a delay time, which are denoted by $\mathcal{P}, \mathcal{D}$, and $\mathcal{T}$, respectively. The probability of the contact represents the reliability of the packet delivery $\mathcal{P}$, the duration of the contact $\mathcal{D}$ represents the capacity of transmission, and the delay of the contact $\mathcal{T}$ affects the its delivery delay.

As discussed in the previous section, SES generates a set of probabilistic state-space graphs, by discretizing the probabilistic contacts. In each statespace graph, the contact duration of each sample is equal to the expected contact duration on the overlapped road, while its probability is equal to the probability of contact. Thus, each edge for a contact is associated with a probabilistic contact duration, termed as probabilistic capacity.

\subsection{Flow Model with Probabilistic Capacities}

The maximum flow in the probabilistic state-space graph is calculated through a flow model with probabilistic capacities [10]. Here, the probabilistic graph can be viewed as a combination of subgraphs, where each subgraph 


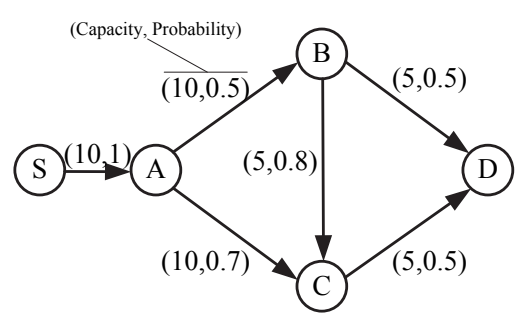

(a) Probabilistic flow in $G$

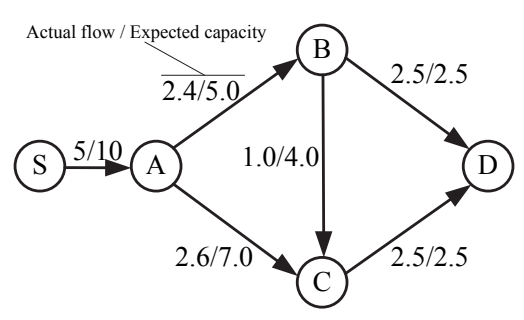

(b) Deterministic flow in $G^{\prime}$

Figure 11: Transform the probabilistic flow into a deterministic flow with upper bound

has a corresponding probability (i.e., the joint probability of the edge capacities). Meanwhile, the maximum flow of each subgraph can be calculated by a classic algorithm (such as the Ford-Fulkerson algorithm). The maximum flow in the probabilistic graph is defined as the mathematical expectation of the maximum flows among these subgraphs.

Let's consider the single commodity flow problem in a two-terminal directed network $N=(G, S, D, c) . G=(V, E)$ is the probabilistic state-space graph, which is a connected directed graph. $S \in V$ denotes the source node, and $D \in V$ denotes the destination node $(S \neq D)$. $c$ denotes a capacity function. Edge failures are assumed to be mutually independent. The failure probability and survival probability of an edge $e \in E$ is denoted by $p(e)$ and $[q(e)=1-p(e)]$, respectively. Let $(N, p)$ denote the resulting probabilistic network, and let $\bar{f}(N, p)$ denote the expected value of the maximum flow in it. As discussed in [10], we consider $\bar{f}(N, p)$ that is subject to random edge failures.

However, calculating the probability distribution of the maximum flow for the probabilistic graph is NP-hard [10]. To approximate the expected maximum flow under the probabilistic networks, we investigate the upper bound and the lower bound of the maximum flow under the networks with probabilistic capacities. The authors in $[18,10]$ have discussed that the upper bound of the maximum flow $\bar{f}(N, p)$ is satisfied by:

$$
\bar{f}(N, p) \leq f(p \cdot N),
$$

where $f(p \cdot N)$ is the maximum flow value in the deterministic network $p \cdot N=$ $\left(G^{\prime}, s, t, c^{\prime}\right) . G^{\prime}\left(V^{\prime}, E^{\prime}\right)$ is a deterministic graph transformed from $G$, where $V^{\prime}=V$ and $E^{\prime}=E$. The capacity function of such deterministic graph $G^{\prime}$ is defined as $c^{\prime}(e)=q(e) c(e)$ for the edge $e \in E^{\prime}$. Therefore, $f(p \cdot N)$ provides an 


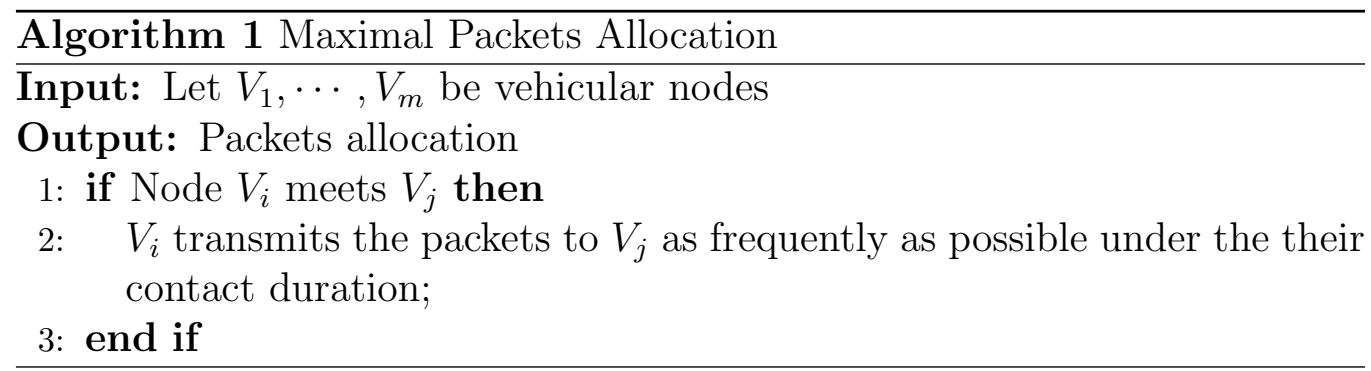

upper bound on $\bar{f}(N, p)$. This means that the upper bound of the maximum flow is equal to the maximum flow of the deterministic networks.

Let $\mathcal{P}(S, D)$ denotes the set of paths from $S$ to $D$ in such network. As discussed in $[18,10]$, the lower bound can be calculated by a feasible flow $h$ along the path $\pi \in \mathcal{P}(S, D)$ as follows:

$$
\bar{f}(N, p) \geq f_{h}(N, p) \equiv \sum_{\pi \in \mathcal{P}(S, D)}\left\{h(\pi) \cdot \prod_{e \in E^{\prime}(\pi)} q(e)\right\} .
$$

To transform the probabilistic model (shown in Figure 11(a)) into a deterministic model, we utilize the upper bound $f(p \cdot N)$ to find a maximum flow in the deterministic graph $G^{\prime}$ shown in Figure 11(b). With the help of the Ford-Fulkerson algorithm, we can calculate the maximum flow on the graph $G^{\prime}$ and obtain the flow on each edge, i.e., $f(e)$ for $e \in E^{\prime}$.

\subsection{Algorithm of Packets Allocation}

Due to the limitation of the bandwidth and contact duration, the original content file is divided into several packets with the same size. For example, the source node $S$ divides a content file into 100 packets. There are 100 packets needed to be delivered from the source node $S$ to the destination node $D$, as shown in Figure 11. During each cycle, the node $A$ receives 10 packets from the source node $S$. Due to the probabilistic contacts with nodes $B$ and $C$, the problem is how to assign the packets to these nodes. We consider three types of assignments:

(1) Maximal packets allocation (Max): as shown in Algorithm 1, when two nodes contact, they transmit the data packets as frequently as possible during their contact. For example, when $A$ contacts with $B$, it will transmit the maximum number of packets according to their contact duration, i.e., 10 packets. However, the simple algorithm does not consider the flow under the network, which may cause the problem of local maximum. For example, there 


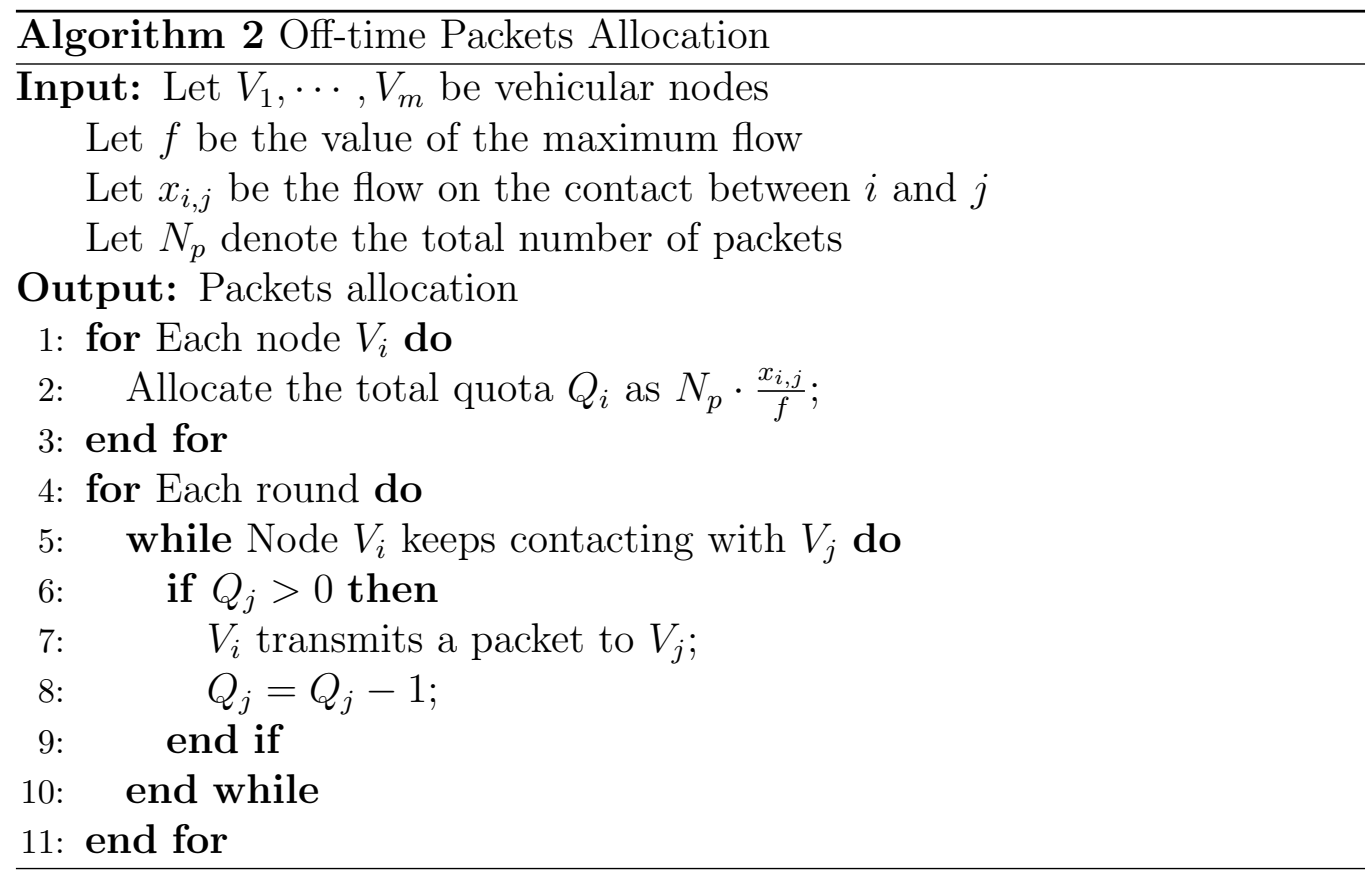

are only two paths from A to $\mathrm{D}$, which are $A \rightarrow B \rightarrow D$ and $A \rightarrow C \rightarrow D$. The capacity of $A \rightarrow B$ is 10 packets, and the capacity of $B \rightarrow D$ is 1 packet. The capacity of $A \rightarrow C$ is 5 packets, and the capacity of $C \rightarrow D$ is 5 packets. If the contact between $\mathrm{A}$ and $\mathrm{B}$ is earlier than the contact between $\mathrm{A}$ and $\mathrm{C}$, many packets could be blocked at $\mathrm{B}$ due to the bottleneck of the contact between $\mathrm{B}$ and $\mathrm{D}$.

(2) Total quota-based packets allocation (Off-time): as shown in Algorithm 2, the source assigns the quota of each node, according to the total number of packets and the flow. When a vehicle contacts another vehicle carrying the packets, the number of the transmitted packets is equal to the minimal value of their contact capacity and the remaining quotas of the vehicle. If a node receives one packet, it will reduce its quotas by 1 . While a node runs out of its quotas, it will no longer receive any packets from the source $S$. For example, B has $100 \cdot \frac{2.4}{2.4+2.6}$ quotas, and C has $100 \cdot \frac{2.6}{2.4+2.6}$ quotas. When A meets $\mathrm{B}$, the number of the packets transmitted from A to $\mathrm{B}$ during this contact is equal to the minimal value between their capacity (i.e., 10 packets) and the remaining quotas of B. Under this algorithm, in the beginning, earlier nodes can receive more packets and consume their quotas faster. When they each run out of their quotas, the later nodes can have 


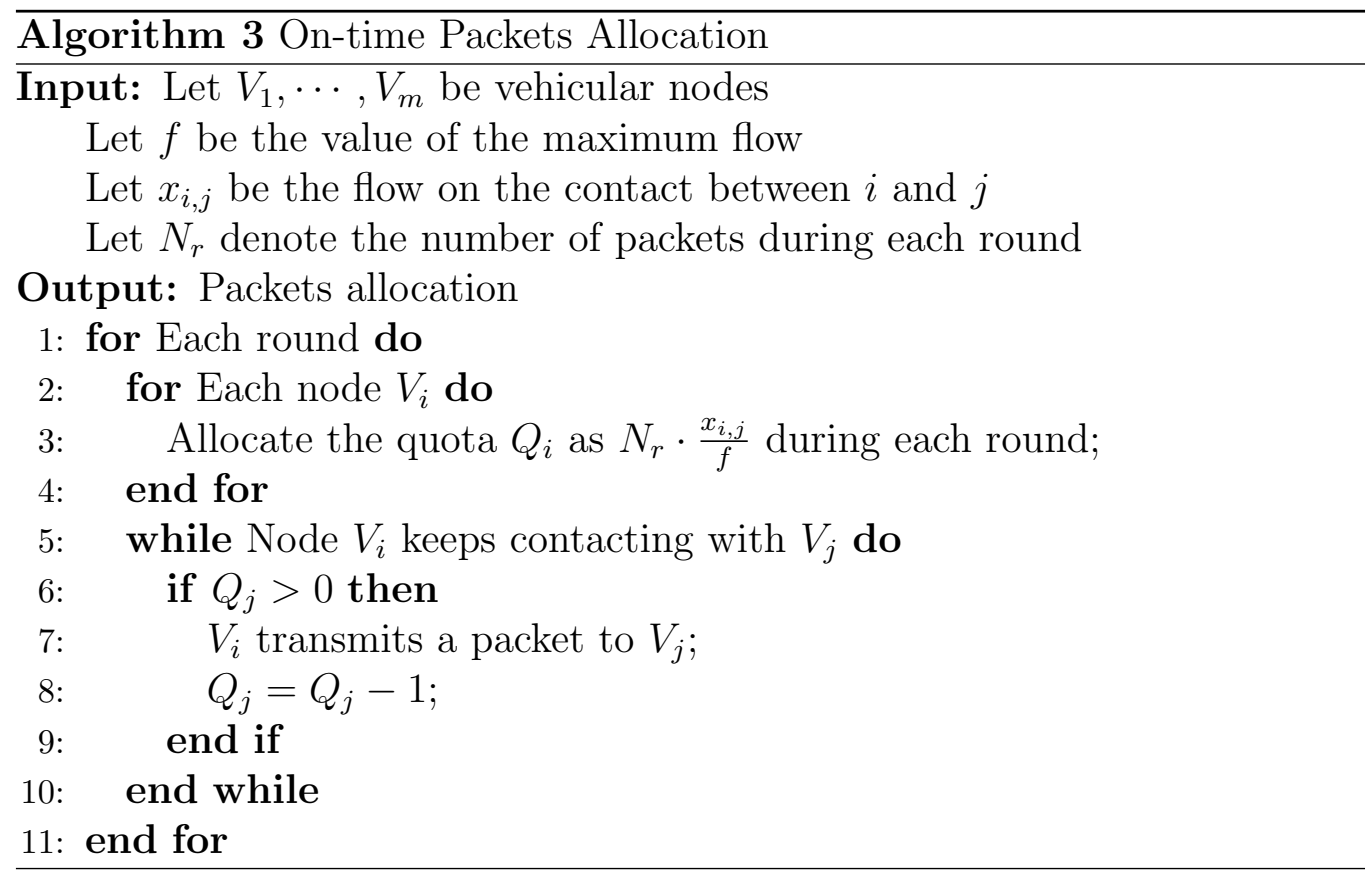

more opportunities for transmitting packets.

(3) Round quota-based packets allocation (On-time): as shown in Algorithm 3, the source assigns the quota of each node based on the number of packets during each round. When a vehicle contacts another vehicle carrying the packets, the number of the transmitted packets is equal to the minimal value between their contact capacity and the remaining quotas of the vehicle. While a node runs out of its quotas, it will no longer receive any packets from the source $S$ during this round. For example, during each round, the vehicle $A$ receives 10 packets from source node. Therefore, during each round, A assigns $10 \cdot \frac{2.4}{2.4+2.6}$ for $\mathrm{B}$, and assigns $10 \cdot \frac{2.6}{2.4+2.6}$ for $\mathrm{C}$. If a node receives one packet, it will reduce its quotas by 1 . When $A$ meets $B$, the number of the packets transmitted from $\mathrm{A}$ to $\mathrm{B}$ during this contact is equal to the minimal value between their capacity (i.e. 10 packets) and the remaining round quotas of B. This algorithm balances the consumption of quotas of different nodes, but under the probabilistic contacts, it may miss many opportunities for content transmission. 


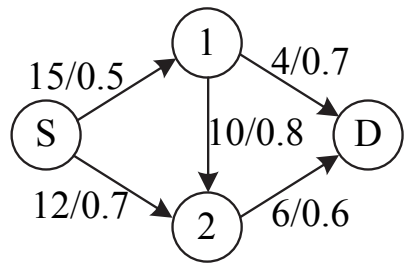

(a) $G$

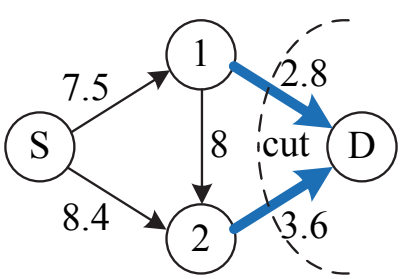

(b) $G^{\prime}$

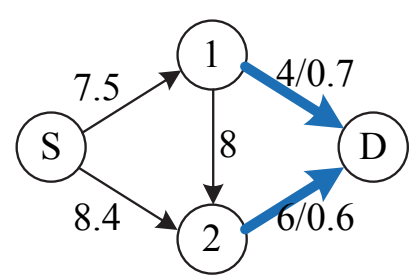

(c) $G^{\prime \prime}$

Figure 12: A simple example for illustrating the algorithm HARM

\subsection{Reliable Content Transmission}

A reliable network is one in which each arc has a certain probability of being functional [19]. Reliable networks are observed in different states, where each state is a conventional network defined on a subset of arcs in the reliable network, which are functional at the point of interest. Since the maximum flows in different states vary, the maximum flow through a reliable network is a random variable, rather than a constant, as in the case of conventional networks.

Expected maximum flows are often computed in reliable networks in order to compare two or more reliable networks defined on the same node set, and to have the same source and terminal nodes. This problem is NP-hard and its solution approaches can be broadly classified into two classes: exact approaches and estimation through Monte Carlo simulations. There is also a literature on generating bounds for this problem.

The problem of reliable content transmission is defined as follows: Given the probability distribution of the maximum flow, we need to determine the flow allocation for content with size $F$ transmitted to the destination; we require this allocation to satisfy that its probability is larger than a threshold called confidence level (denoted by $\delta$ ), i.e. $P[f \geq F] \geq \delta$. Again, $P[X]$ denotes the probability of $X$ is true, and so $P[f \geq F]$ represents the probability of the maximum flow $f$, which is larger than its allocation $F$. During the content transmission, when a vehicle $A$ meets $B$, it makes a decision of allocating the content $F$ for the two vehicles $\left(F_{A}\right.$ and $\left.F_{B}\right)$. Let $f_{A}$ denote the maximum flow from the vehicle $A$ to the destination, and the allocation decision is based on the maximum flows with the two vehicles as the sources, 


\begin{tabular}{l}
\hline Algorithm 4 HARM \\
Input: Probabilistic graph $G(V, E)$ \\
Output: PDF of max-flow \\
1: Calculate the expected capacities of edges in $E^{\prime}$ \\
2: Obtain a deterministic graph $G^{\prime}\left(V^{\prime}, E^{\prime}\right)$ \\
3: Find the minimum cut in $G^{\prime}$ by marking the edges \\
4: Recover the probabilistic capacities of marked edges \\
5: Generate a mixed graph $G^{\prime \prime}\left(V^{\prime}, E^{\prime \prime}\right)$ \\
6: Calculate PDF of max-flow with marked edge in $G^{\prime \prime}$ \\
7: return PDF of max-flow \\
\hline
\end{tabular}

respectively. The optimization problem is defined as follows:

$$
\begin{array}{ll}
\underset{F_{A}, F_{B}}{\operatorname{maximize}} & P\left[f_{A} \geq F_{A}\right] \cdot P\left[f_{B} \geq F_{B}\right] \\
\text { s.t. } & P\left[f_{A} \geq F_{A}\right] \geq \delta, \\
& P\left[f_{B} \geq F_{B}\right] \geq \delta, \\
& F_{A}+F_{B}=F .
\end{array}
$$

This problem can be solved through flow capacity scheduling, i.e., discretize $f_{A}, f_{B}, F_{A}$, and $F_{B}$. For example, if $F=10$, we can solve these variables by approximating them to be integers in the interval between 1 and 10 , and then compare all combinations of $F_{A}$ and $F_{B}$. If $F$ is discretized to $\Delta$ stages, the time complexity of solving the above problem is $O\left(\Delta^{2}\right)$. Since capacity scheduling has been discussed in many works [20], here, we do not further discuss its approximation performance.

Since calculating the probability distribution of the maximum flow for the probabilistic graph is NP-hard [10], we propose a Heuristic Algorithm for the Reliability of Max-flow (HARM). Given a probabilistic state-space graph $G(V, E)$, HARM is shown in Algorithm 4. Figure 12(a) shows an example of a probabilistic state-space graph $G$ for calculating the maximum flow from $S$ to $D$ with the algorithm HARM. We obtain a deterministic graph $G^{\prime}\left(V^{\prime}, E^{\prime}\right)$ shown in Figure 12(b) from the probabilistic graph $G$, where the capacity of each edge in $E^{\prime}$ is equal to the expected capacity of its corresponding edge in $E$. Then, we calculate a minimum cut in the deterministic graph $G^{\prime}$, and mark the edges on the cut, as the thick edges in Figure 12(b). We obtain a mixed graph $G^{\prime \prime}\left(V^{\prime}, E^{\prime \prime}\right)$ by recovering the capacity of each marked edge as the probabilistic capacity in $G$, and keeping the capacity of each unmarked 


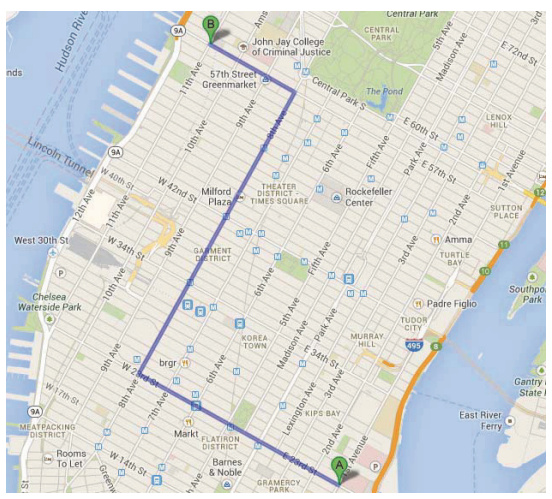

(a) Manhattan streets in NYC

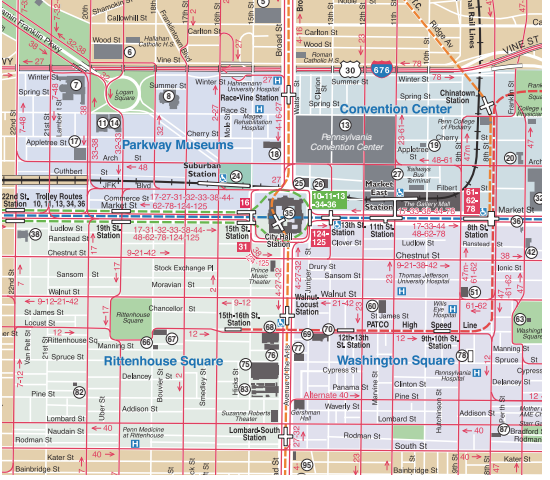

(b) SEPTA system in Philadelphia

Figure 13: Maps used in Simulations

edge as their expected value, as shown in Figure 12(c). Thus, we calculate the probability density function (PDF) of the maximum flow in $G^{\prime \prime}$ by combining the capacities of the marked edges, as the approximated PDF of maximum flow in the probabilistic graph $G$. In the example, the four maximum flows are $10,6,4$, and 0 , whose probabilities are $0.42,0.18,0.28$, and 0.12 .

\section{Performance Evaluation}

In this section, we evaluate the performance of routing and content transmission with our proposed Sampling-based Estimation Scheme (SES). First, we introduce the methodology used in our evaluations. Then, we present the results of performance compared with other protocols.

\subsection{Methodology}

For evaluating the performance of algorithms under different conditions, we use two types of traces. One type of trace is generated by SUMO (Simulation of Urban Mobility) [12], which is a well-known open-source traffic simulator to model realistic vehicle behavior. The speed of vehicles in the trace generated by SUMO follows a Gaussian distribution of $N\left(\mu_{v}, \sigma_{v}\right)$. We use a combination of MOVE [13], SUMO, and NS-2 [11], which we term as $M S N$, for the simulations of VANETs. MOVE is an extension to SUMO that allows its vehicle mobility traces to be imported into NS-2. NS-2 is an open-source discrete event network simulator that supports both wired and 
Table 3: Simulation parameters

\begin{tabular}{|l||l|}
\hline Parameter & NS-2 Setting Value \\
\hline \hline Channel type & Channel/WirelessChannel \\
\hline Radio propagation model & Propagation/TwoRayGround \\
\hline Network interface type & Phy/WirelessPhy \\
\hline MAC type & 802.11 \\
\hline Interface queue type & Queue/DropTail/PriQueue \\
\hline Link layer type & LL \\
\hline Antenna model & Antenna/OmniAntenna \\
\hline
\end{tabular}

wireless networks, including most MANET routing protocols and an implementation of the IEEE 802.11 MAC layer.

Under the MSN-based simulations, we use the road topology of Manhattan streets in New York City, as shown in Figure 13(a). We choose the area of 1.7 mile $\times 1.7$ mile in Manhattan with 100 intersections $(10 \times 10)$. The average length of each road segment is 0.2 mile. Five APs are uniformly distributed in the area and there are 20 vehicles.

Inspired by the DieselNet bus trace [9] and MobiSpace [8], we generate a set of synthetic bus traces from maps and schedules of the SEPTA system in Philadelphia [5], as shown in Figure 13(b). We develop a tool to extract the routes from the maps and schedules of buses. The speed of vehicles in the trace also follows a Gaussian distribution of $N\left(\mu_{v}, \sigma_{v}\right)$.

The communication range of each vehicle $(R)$ is 100 meters and the bandwidth is $5 \mathrm{Mb} / \mathrm{s}$. The source of data delivery is a randomly chosen vehicle to generate a data packet to the destination, which is one of the APs. SES is used to find an optimal routing path with expected minimum delay. With probabilistic contact durations, our SES-based content transmission scheme can find the reliable maximum flow in the networks, which cannot only guarantee the reliability of content transmission, but also reduce its delay during each cycle. For comparison with other algorithms, we choose to evaluate them according to the following two important metrics: (1) Data delivery delay: the duration of the data delivery from the moment it is generated by the source to the arrival time at the destination; (2) Data arrival ratio: the ratio between the portion of the data received by the sink at the final destination and the total amount of the data originated by the source.

\subsection{Performance of Routing}

In this subsection, we evaluate the performance of routing by the proposed scheme SES with two samples. The protocols chosen for evaluating 


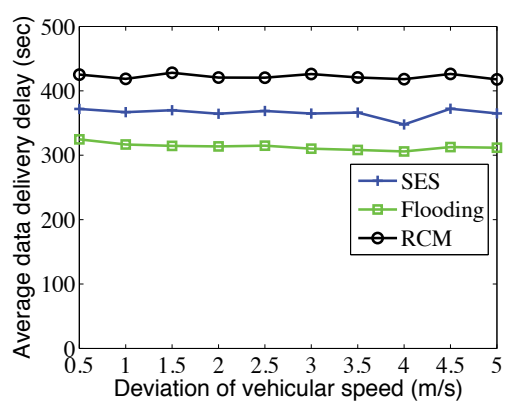

(a) Speed of $5 \mathrm{~m} / \mathrm{s}$

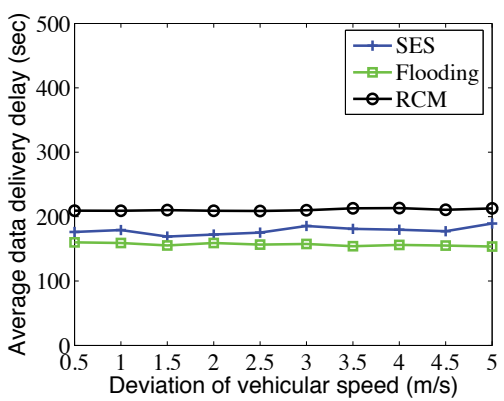

(b) Speed of $10 \mathrm{~m} / \mathrm{s}$

Figure 14: Data delivery delay in Manhattan

include: (1) RCM in [8] which discretizes each probabilistic contact between two nodes as a point associated with a time slot and a contact probability; (2) Flooding: we assume there is no transmission conflict under a sparse node distribution, and vehicles have an infinite buffer to store packets, based on which a vehicle simply forwards packets to every other vehicle it meets. With these assumptions, the flooding protocol achieves minimal delivery delay.

We compare the performance of the three protocols by the MSN-based simulations with the road topology of Manhattan. Figure 14(a) shows the results of the average data delivery delay under different derivations of vehicular speed $\sigma_{v}$. The average speed of vehicles is $5 \mathrm{~m} / \mathrm{s}$. Due to the assumption of the infinite buffer and collision-free, flooding can achieve the minimal delay. The data delivery of SES-based routing protocols is lower than that of RCM. This is because the estimation with the contacts by the proposed protocol SES has more available routing paths and higher accuracy than the RCM. Figure 14(b) shows the results of the average data delivery delay under different derivations of vehicular speed $\sigma_{v}$, and the average speed of each vehicle is $10 \mathrm{~m} / \mathrm{s}$. Under the higher vehicular speed, the data delivery delays with different protocols are reduced. We notice that the delay with SES is much lower than that of RCM and is also closer to flooding.

Meanwhile, we also compare the performance of the three protocols with the bus traces from maps and schedules of the SEPTA system in Philadelphia [5]. Due to the fixed schedules of buses on their routes, the average speed is fixed. We choose two groups of buses: The first group includes 10 buses that have a short duration of each cycle (less than 60 minutes), and the second group includes 10 buses that have a long duration of each cycle (more than 


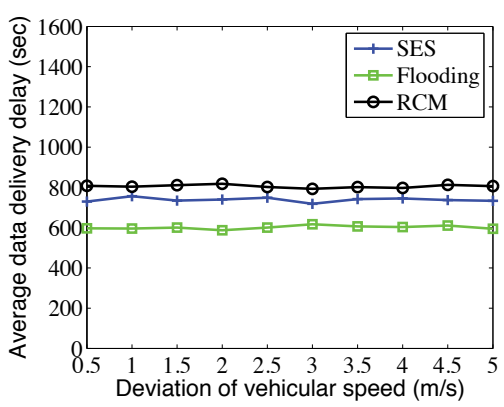

(a) Short cycle

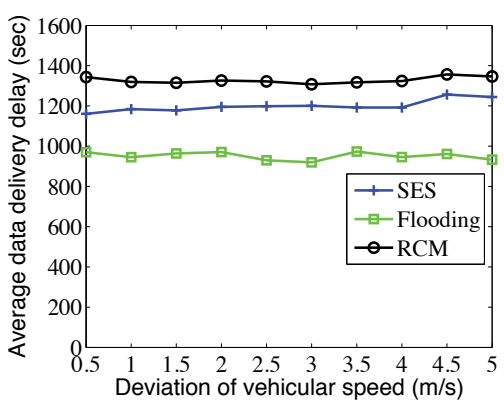

(b) Long cycle

Figure 15: Data delivery delay with SEPTA

60 minutes). Five APs are uniformly placed in the area. Figures 15(a) and 15(b) show the results of the data delivery delay of the buses in the short cycle group and long cycle group, respectively. We notice that flooding has the minimal delay with different derivations of vehicular speed. The average delay obtained by SES-based routing is lower than that under RCM.

\subsection{Performance of Content Transmission}

For evaluating the performance of our proposed SES-based reliable content transmission scheme, two protocols are chosen as the benchmarks as follows: (1) Flooding: when a vehicle contacts another vehicle, it transmits the maximal amount of data within the constraint of their contact duration; (2) Binary spray: when a vehicle contacts another vehicle, it transmits half of the amount of the content data which it carries to the contacting vehicle. The setup of the simulations is the same as above.

We compare the three protocols with MSN-based simulations to the map of Manhattan. Figure 16(a) shows the results of the content arrival ratio under different demands during one cycle of the vehicles' trajectories. The derivations of vehicular speed is $3 \mathrm{~m} / \mathrm{s}$. The protocol of flooding only considers the duration of current contact, which does not consider the maximum flow from the contacting vehicle to the destination. Thus, it will cause the loss of content, if the duration of one of the next several contacts is less than the current one. When the demand is small, the amount of transmitting data during a contact is less than the capacity, and it causes a slight increase. While the amount is larger than the capacity, it will cause the loss of content and reduce its arrival ratio. Binary spray does not utilize the maximum flow 


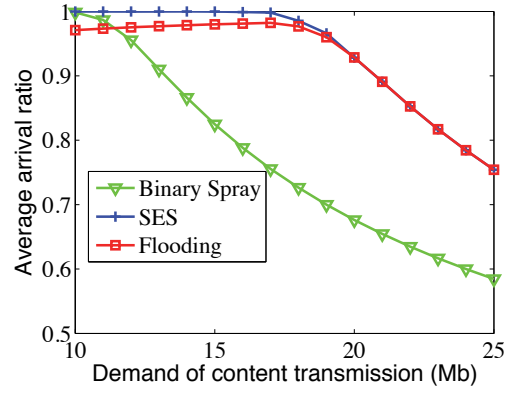

(a) $\sigma=3$

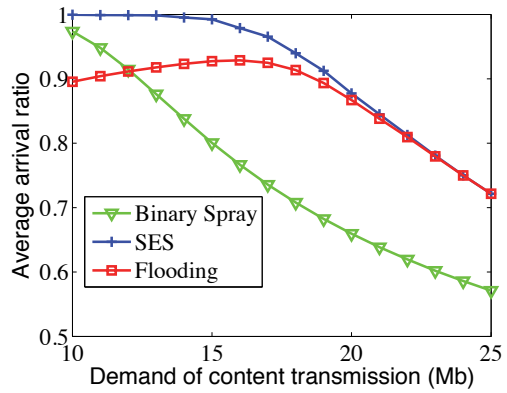

(b) $\sigma=7$

Figure 16: Arrival ratio of content transmission in Manhattan

of each branch to the destination, so it has the lowest arrival ratio while the demand is increasing. The proposed SES-based algorithm has the highest arrival ratio, even when the demand is larger than the maximum flow of the vehicles. Figure 16(b) shows the results in which derivations of vehicular speed is $7 \mathrm{~m} / \mathrm{s}$. Our proposed algorithm also has the highest arrival ratio. We notice that the arrival ratio of content approaches 1 by SES under a small value of demand for content transmission. This is because a small demand has a higher reliability of transmission with maximum flow.

The large size of content transmission needs to be transmitted during several rounds with cyclic trajectories (such as buses). Our SES-based scheme transmits content with a high probability of maximum flow, so it can reduce the number of rounds. We evaluate the delay of content transmission with the trace of SEPTA compared with the other two protocols, i.e., flooding and RCM. In RCM, the content is always transmitted along the shortest routing path. Figure 17(a) shows the results of the content transmission delay with different demands, while derivations of vehicular speed is $3 \mathrm{~m} / \mathrm{s}$. The protocol of flooding only considers the duration of current contact, which does not consider the content transmission delay along the flow from each branch to the destination. Thus, it will cause higher content transmission delay by some branches with higher delay. RCM does not utilize multiple flows to the destination, so it also has higher delay while the demand increases. The proposed SES-based algorithm has the lowest content transmission delay while the demand increases. Figure 17(b) shows the results under the case in which derivations of vehicular speed is $7 \mathrm{~m} / \mathrm{s}$. We notice that the SES-based algorithm also has the lowest content transmission delay. 


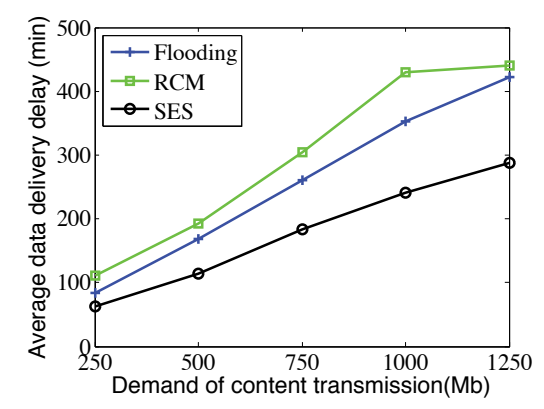

(a) $\sigma=3$

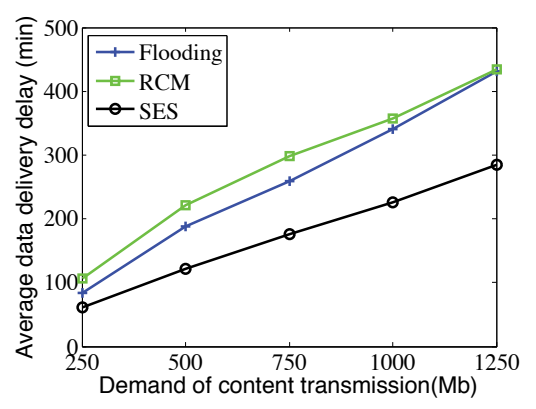

(b) $\sigma=7$

Figure 17: Average delivery delay of content transmission with SEPTA

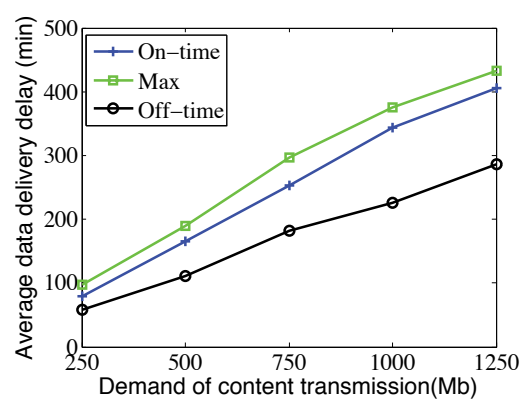

(a) $\sigma=3$

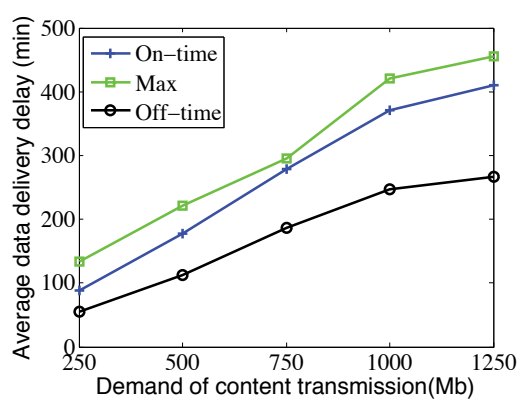

(b) $\sigma=7$

Figure 18: Average delivery delay of content transmission with SEPTA

We evaluate the performances of different schemes for content transmission, which are Max, On-time, and Off-time, with MSN-based simulations and the trace of SEPTA. Figure 18(a) shows the results of the content transmission delay with different demands, while derivations of vehicular speed is $3 \mathrm{~m} / \mathrm{s}$. Figure 18(b) shows the results under the case in which derivations of vehicular speed is $7 \mathrm{~m} / \mathrm{s}$. We notice that the average delivery delay is increasing, while the demand of the content transmission is increasing. The protocol of Max only considers the duration of current contact, which does not consider the content transmission delay along the flow from each branch to the destination. Thus, it will cause higher content transmission delay by some branches with higher delay. The protocol of off-time has the lowest data delivery delay. 


\section{Related Work}

Many vehicular applications have become available based on the vehicleto-vehicle communications. Ahn et al. [21] present the road information sharing architecture, a distributed approach to road condition detection and dissemination for vehicular networks. SignalGuru [22] relies solely on a collection of mobile phones to detect and predict the traffic signal schedule, where multiple phones in the vicinity use opportunistic ad-hoc communications to collaboratively learn the timing patterns of traffic signals, and then to predict their schedules. MARVEL [23] utilizes the communications among vehicles to determine their relative locations. Forster et al. in [24] present a approach (CADAS) which connects vehicles via VANETs and lets them exchange recent and relevant traffic information, to eliminate upstream shock wave formation even with low system penetration rates. $\mathrm{Hu}$ et al. in [25] propose a fully deployed smartphone-based vehicular mobile sensing system in which automatic phone-to-phone communication is achieved and is compatible with existing wireless infrastructure.

In [26], authors describe the pair-wise contacts as follows: Pair-wise contacts between users/devices can be characterized by the means of two parameters: contact durations and inter-contact times. The duration of a contact is the total time that a tagged couple of mobile nodes are within reach of each other, and thus have the possibility of communicating. An inter-contact time is, instead, the time between two contact opportunities between the same couple of tagged devices. While the contact duration directly influences the capacity of opportunistic networks, because it limits the amount of data that can be transferred between nodes, the inter-contact time affects the feasibility of opportunistic networks, and the delay associated with them.

Many studies have investigated the contact-based forwarding scheme with the knowledge of vehicles' trajectories. Jeong et al. in [27] investigate how to effectively utilize vehicles' trajectory information in a privacy-preserving manner, where the trajectory information is used to improve the performance of data forwarding in road networks. Xu et al. in [28] propose a data forwarding scheme, which utilizes shared vehicle trajectory information. For calculating multi-hop encounter events, they approximate each encounter event, based on the previous encounter as one point on the road. Zhu et al. in [4] study three large sets of Global Positioning System (GPS) traces of more than ten thousand public vehicles, collected from Shanghai and Shenzhen. By mining the temporal correlation and evolution of contacts between 
each pair of vehicles, they use higher order Markov chains to characterize urban vehicular mobility patterns. Wu et al. in [7] show that there is strong spatio-temporal regularity with vehicle mobility, and they also develop multiple order Markov chains for predicting future trajectories of vehicles. Then, the next hop for message forwarding is determined based on the previous contacts. Zhu et al. in [4] use higher order Markov chains to characterize urban vehicular mobility patterns, by mining the temporal correlation and the evolution of contacts between each pair of vehicles.

The content transmission in VANETs, such as media applications, is based on the contact durations among vehicles, which typically boils down to the consecutive transmissions of content files from the source to the destination. Luan et al. in [29] focus on provisioning the efficient media applications in highway vehicular networks through the framework of integrity-oriented content transmissions; in order to ensure the successful presentation of ontop media applications, digital contents are required to be fully transmitted in their entirety.

\section{Conclusion}

Data delivery in VANETs is based on the contacts among the vehicles. Contacts among vehicles happen on the overlapped roads of their trajectories. Due to the stochastic vehicular mobility, the contacts on the overlapped roads are probabilistic. The contacts on the overlapped roads play an important role of routing in VANETs, due to the high probability and long duration of the contact on it. In this paper, we investigated the probabilistic contacts on the overlapped roads of the trajectories, which have high spatio-temporal fluctuation. Thus, we propose a novel Sampling-based Estimation Scheme (SES) by leveraging the accuracy and computational cost. Moreover, the contact durations are also stochastic, which affects the throughput between two inter-contact vehicles. For the content transmission, we investigate maximum flow among the probabilistic contacts, through the flow model with probabilistic capacities. For the probabilistic duration of contact, we propose a reliable content transmission scheme with a probabilistic flow model, and propose a heuristic algorithm HARM for approximately estimating the distribution of maximum flow. We conduct intensive simulations for evaluating the performance of SES for the probabilistic contacts on the overlapped roads in VANETs. 


\section{Acknowledgment}

This work is supported by NSF grants ECCS 1231461, ECCS 1128209, CNS 1138963, CNS 1065444, and CCF 1028167; NSFC grants No. 61003229, 61170256, 61103226, 61173172, 61272526, 61370204; and the Fundamental Research Funds for the Central Universities No. ZYGX2009J063, ZYGX2013J077, ZYGX2013J067; and the Applied Basic Program of Sichuan Province of China No. 2014JY0192.

\section{References}

[1] S. Zeadally, R. Hunt, Y.-S. Chen, A. Irwin, A. Hassan, Vehicular ad hoc networks (vanets): status, results, and challenges, Telecommunication Systems 50 (4) (2012) 217-241.

[2] J. Zhao, G. Cao, Vadd: Vehicle-assisted data delivery in vehicular ad hoc networks, IEEE Transactions on Vehicular Technology 57 (3) (2008) 1910-1922.

[3] J. Wu, S. Yang, Polylogarithmic store-carry-forward routing using mobile nodes, in: Proc. of IEEE MASS, 2007.

[4] H. Zhu, S. Chang, M. Li, K. Naik, S. Shen, Exploiting temporal dependency for opportunistic forwarding in urban vehicular networks, in: Proc. of IEEE INFOCOM, 2011.

[5] Septa.

URL www.septa.org

[6] I. Leontiadis, P. Costa, C. Mascolo, Extending access point connectivity through opportunistic routing in vehicular networks, in: Porc. of IEEE INFOCOM, 2010.

[7] Y. Wu, Y. Zhu, B. Li, Trajectory improves data delivery in vehicular networks, in: Proc. of IEEE INFOCOM, 2011.

[8] C. Liu, J. Wu, Routing in a cyclic mobispace, in: Proc. of ACM MobiHoc, 2008.

[9] X. Zhang, J. Kurose, B. N. Levine, D. Towsley, H. Zhang, Study of a bus-based disruption-tolerant network: mobility modeling and impact on routing, in: Proc. of ACM MobiCom, 2007. 
[10] H. Nagamochi, T. Ibaraki, Maximum flows in probabilistic networks, Networks 21 (6) (1991) 645-666.

[11] The network simulator - ns-2.

URL http://isi.edu/nsnam/ns/

[12] Sumo - simulation of urban mobility.

URL http://sumo. sourceforge.net/

[13] F. K. Karnadi, Z. H. Mo, K.-c. Lan, Rapid generation of realistic mobility models for vanet, in: Proc. of WCNC, 2007.

[14] A. Polus, A study of travel time and reliability on arterial routes, Transportation 8 (2) (1979) 141-151.

[15] R. P. Roess, E. S. Prassas, W. R. McShane, Traffic engineering, Pearson/Prentice Hall, 2004.

[16] M. J. Khabbaz, W. F. Fawaz, C. M. Assi, A probabilistic and trafficaware bundle release scheme for vehicular intermittently connected networks, IEEE Transaction on Communications 60 (11).

[17] A. Ferreira, Building a reference combinatorial model for manets, IEEE Network 18 (5) (2004) 24-29.

[18] M. Carey, C. Hendrickson, Bounds on expected performance of networks with links subject to failure, Networks 14 (3) (1984) 439-456.

[19] M. Sharma, D. Ghosh, Speeding up the estimation of expected maximum flows through reliable networks, Indian Institute of Management, 2009.

[20] J. Kleinberg, E. Tardos, Algorithm design, Pearson Education India, 2006.

[21] J. Ahn, Y. Wang, B. Yu, F. Bai, B. Krishnamachari, Risa: Distributed road information sharing architecture, in: Proc. of IEEE INFOCOM, 2012.

[22] E. Koukoumidis, L.-S. Peh, M. R. Martonosi, Signalguru: leveraging mobile phones for collaborative traffic signal schedule advisory, in: Proc. of ACM MobiSys, 2011. 
[23] D. Li, T. Bansal, Z. Lu, P. Sinha, Marvel: Multiple antenna based relative vehicle localizer, in: Proc. of ACM Mobicom, 2012.

[24] M. Forster, R. Frank, M. Gerla, T. Engel, A cooperative advanced driver assistance system to mitigate vehicular traffic shock waves, in: Proc. of IEEE INFOCOM, 2014.

[25] S. Hu, H. Liu, L. Su, H. Wang, T. F. Abdelzaher, P. Hui, W. Zheng, Z. Xie, J. A. Stankovic, Towards automatic phone-to-phone communication for vehicular networking applications, in: Proc. of IEEE INFOCOM, 2014.

[26] L. Pelusi, A. Passarella, M. Conti, Opportunistic networking: data forwarding in disconnected mobile ad hoc networks, IEEE Communications Magazine 44 (11) (2006) 134-141.

[27] J. Jeong, S. Guo, Y. Gu, T. He, D. Du, Tbd: Trajectory-based data forwarding for light-traffic vehicular networks, in: Proc. of IEEE ICDCS, 2009.

[28] F. Xu, S. Guo, J. Jeong, Y. Gu, Q. Cao, M. Liu, T. He, Utilizing shared vehicle trajectories for data forwarding in vehicular networks, in: Proc. of IEEE INFOCOM, 2011.

[29] T. H. Luan, X. S. Shen, F. Bai, Integrity-oriented content transmission in the vehicular ad hoc networks, in: Proceedings of IEEE INFOCOM, 2013. 